\title{
Myelopathy and Quadriparesis due to Spinal Cord Compression of C1 Laminar Osteochondroma
}

\author{
Uygur Er ${ }^{1}$, Serkan Şimşek ${ }^{1}$, Kazım Yiğitkanlı ${ }^{1}$ Aysegül Adabağ ${ }^{2}$, Hamit Zafer Kars ${ }^{1}$ \\ ${ }^{1}$ Second Neurosurgery Clinic, Yıldırım Beyazıt Education and Research Hospital, Diskapi, Ankara, Turkey \\ ${ }^{2}$ Department of Pathology, Yıldırım Beyazıt Education and Research Hospital, Diskapi, Ankara, Turkey
}

\begin{abstract}
The aim of this paper is to show that osteochondromas of the cervical vertebrae can cause myelopathy and neck pain. The reported etiology, diagnosis, treatment and differential diagnosis were reviewed. Osteochondromas may present as a solitary lesion with no genetic component or as multiple lesions as a part of a genetic disorder known as hereditary multiple exostosis. Osteochondromas of the spine are rarely encountered in clinical practice. These lesions are reported more commonly with neural compression in cases associated with hereditary multiple exostosis. The authors describe a unusual clinical manifestation of a solitary osteochondroma located in the right posterior arch of the atlas. Complete removal of the tumor was performed resulting in the relief of neck pain and spastic quadriparesis. Although unusual,osteochondromas of the cervical spine must be considered in patients with persistent neck pain and progressive symptoms of myelopathy. Computed tomography and magnetic resonance imaging in conjunction with plain radiograms is the neuroradiological modality of choice. The diagnosis and surgical excision of these tumors are important because they can cause spinal stenosis resulting in neural tissue compression and myelopathy.
\end{abstract}

Key Words: Atlas, C1, Cervical vertebrae,Spinal cord diseases, Quadriparesis, Solitary osteochondroma

\section{Introduction}

Osteochondromas are the most common benign tumors of the appendicular skeleton. The pathology consists of progressive enchondral ossification from the aberrant cartilage of a growth plate that makes the long bones the most affected localization [1]. They occur less frequently in the axial skeleton, and an association with compression of neural elements is quite rare.

Osteochondromas may present as a solitary lesion with no genetic component or as multiple lesions in the form of the genetic disorder known as hereditary multiple exositosis (HME). HME has a higher incidence of spinal involvement (3\% of cases) and neurological complications than the solitary variety. The occurrence of osteochondromas, particularly in cases of HME, is generally in young adults with most cases becoming symptomatic during the second and third decades of life [2]. The progression of these symptoms has been associated with continued bone growth.

We encountered an unusual case of a solitary osteochondroma arising from the posterior arch of atlas, which was signaled by progressive neck pain and symptoms of myelopathy. This case is reported with a review of the relevant literature.

\section{Case Report}

A 42-year-old woman was admitted to hospital with the complaints of weakness, pain and numbness in all extremities that had lasted for 4 months, and a 10-year

Received Oct 18, 2010; Revised Nov 16, 2010; Accepted Nov 18, 2010

Corresponding author: Uygur Er, MD

Second Neurosurgery Clinic, Yıldırım Beyazıt Education and Research Hospital,

Söğütözü C., 4th Sk., No. 22/7, 06510, Çankaya, Ankara, Tukey

Tel: +90-505-589-23-55, Fax: +90-312-517-31-44, E-mail: uygurer@gmail.com 
history of persistent neck pain. Her pain was mainly in the suboccipital area and radiated to the right retroauricular region. Her symptoms increased gradually with time. Her medical history revealed that she had fallen from a wall onto a concrete ground 10 years earlier and hit her head. Since then, she had neck pain exaggerated by motion.

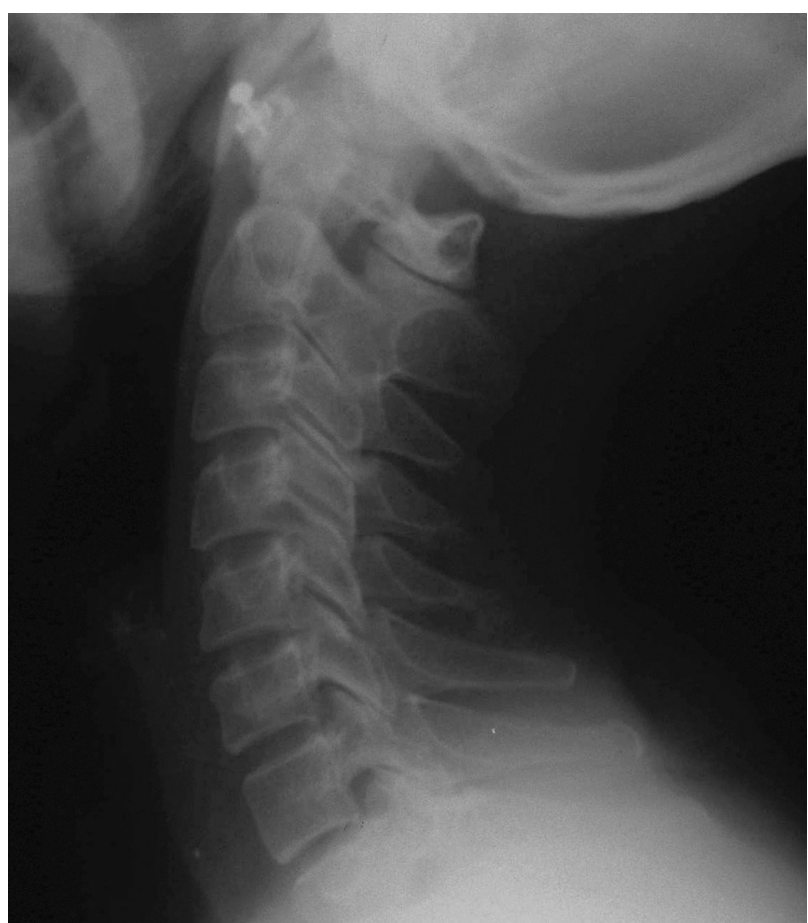

Fig. 1. Lateral cervical radiograph showing a bony lesion in the posterior arch of the atlas.

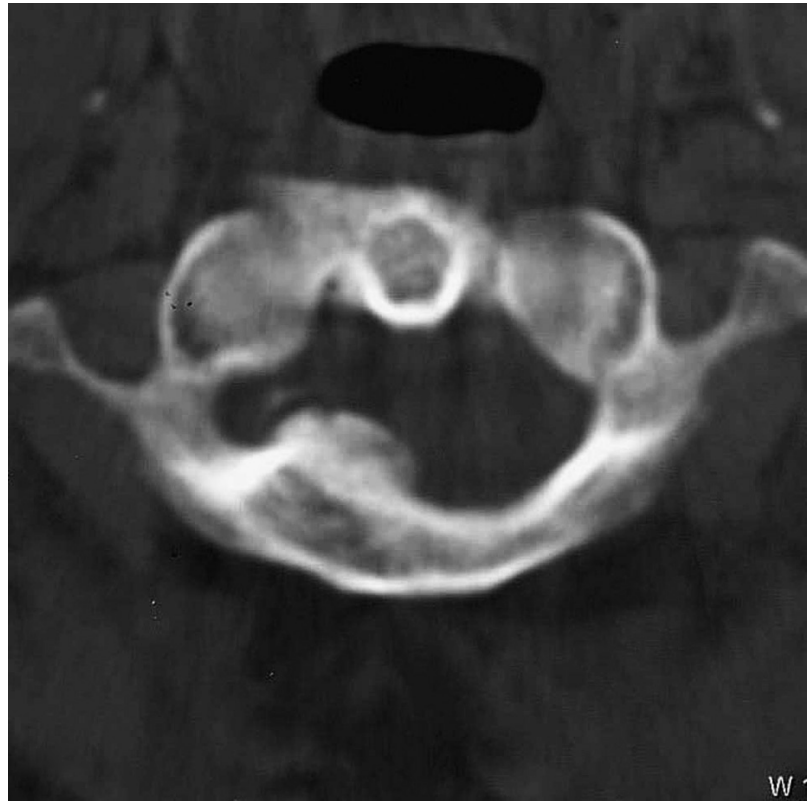

Fig. 2. Bone window axial computed tomography scan at the level of $\mathrm{C} 1-2$ showing an abnormal bony mass arising from the inner side of the posterior arch of $\mathrm{C} 1$ at the right site.
Various medical treatments were attempted without a clear diagnosis. Previous radiological examinations were unavailable. Upon admission, the neurological examination

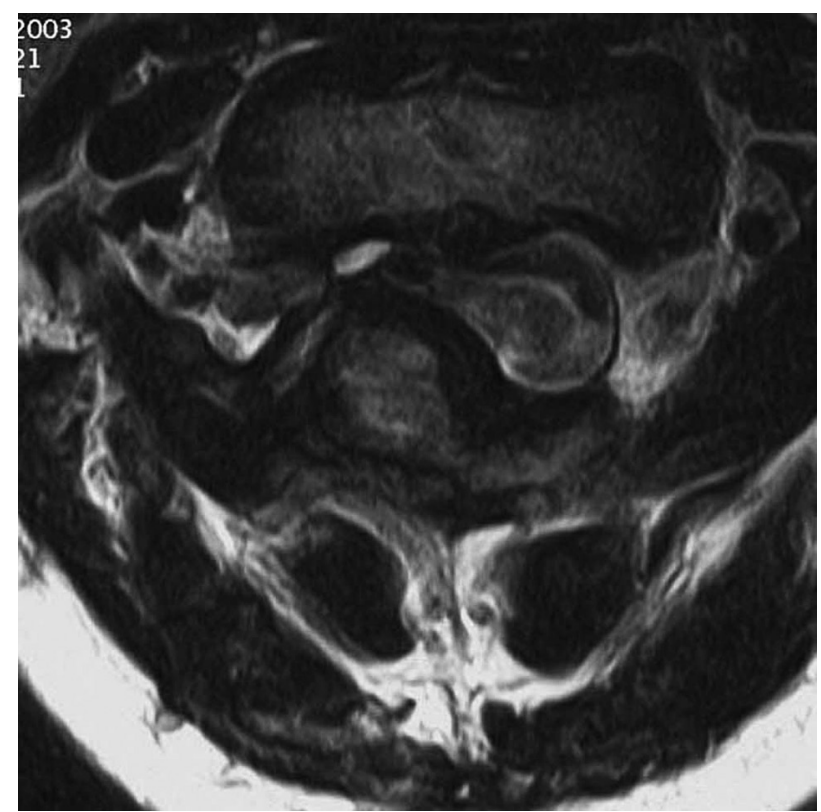

Fig. 3. Axial T1-weighted magnetic resonance imaging revealing a bony mass in the posterior arch of the atlas and a hyperintense lesion is visible in the cord.

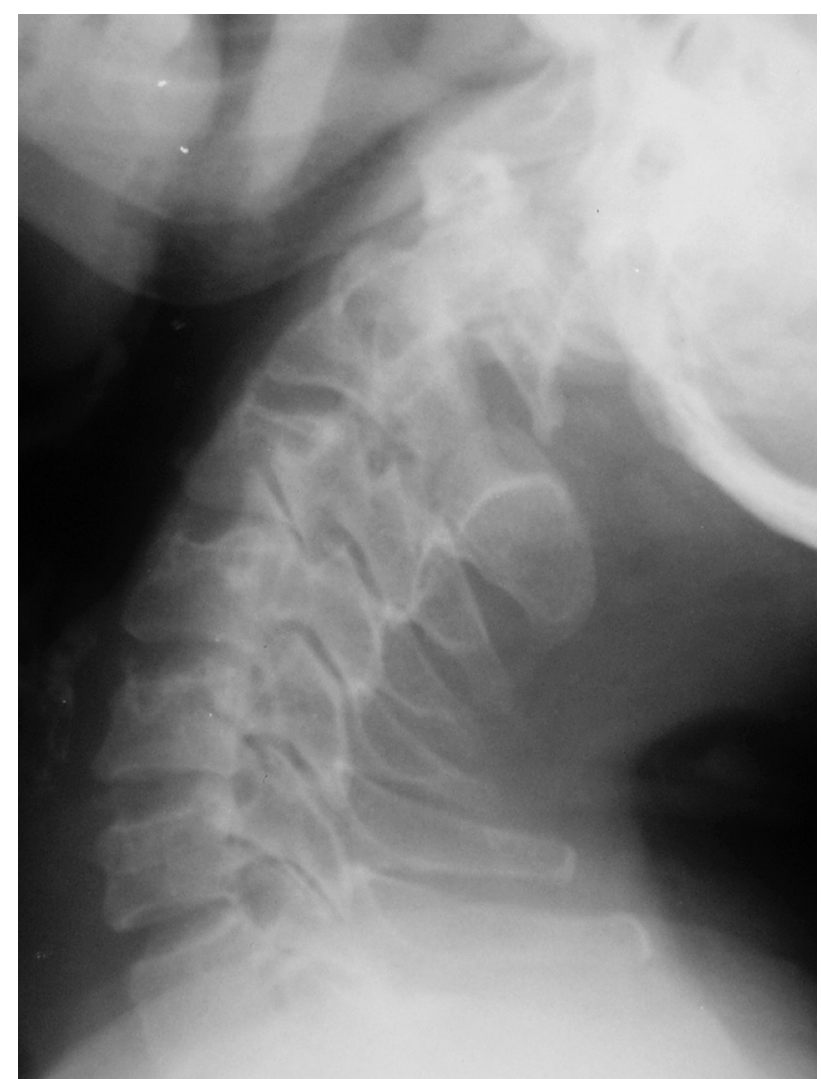

Fig. 4. Postoperative lateral radiograph showing the removal of the bony lesion. 
revealed spastic quadriparesis (in 3/5 muscle strength) and hypoesthesia below the $\mathrm{C} 2$ level. The deep tendon reflexes were hyperactive in all extremities. Clonus was elicited at both ankles and knees with bilateral extensor plantar responses. An evaluation of the severity of myelopathy using the Japan Orthopedic Association (JOA) scale gave a score of 10 out of 18 . A lateral cervical radiogram revealed a hypertrophic lesion in the posterior arch of the first vertebra (Fig. 1). Computed tomography (CT) revealed bony outgrowth from the right anterior aspect of the posterior arch of $\mathrm{Cl}$ (Fig. 2). Magnetic resonance imaging (MRI) confirmed the presence of a homogenous irregular contoured bony mass, compressing the spinal cord with

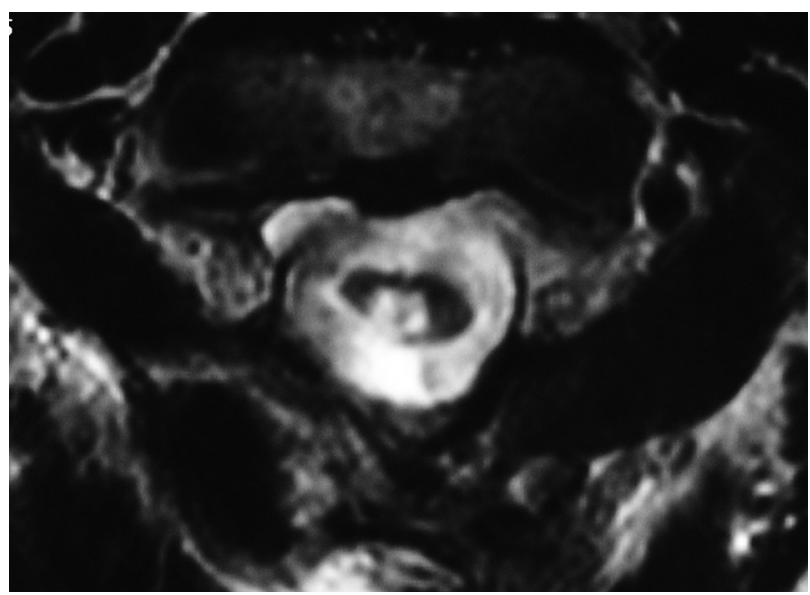

Fig. 5. Postoperative axial T2-weighted magnetic resonance imaging showing no visible lesion in the spinal cord.

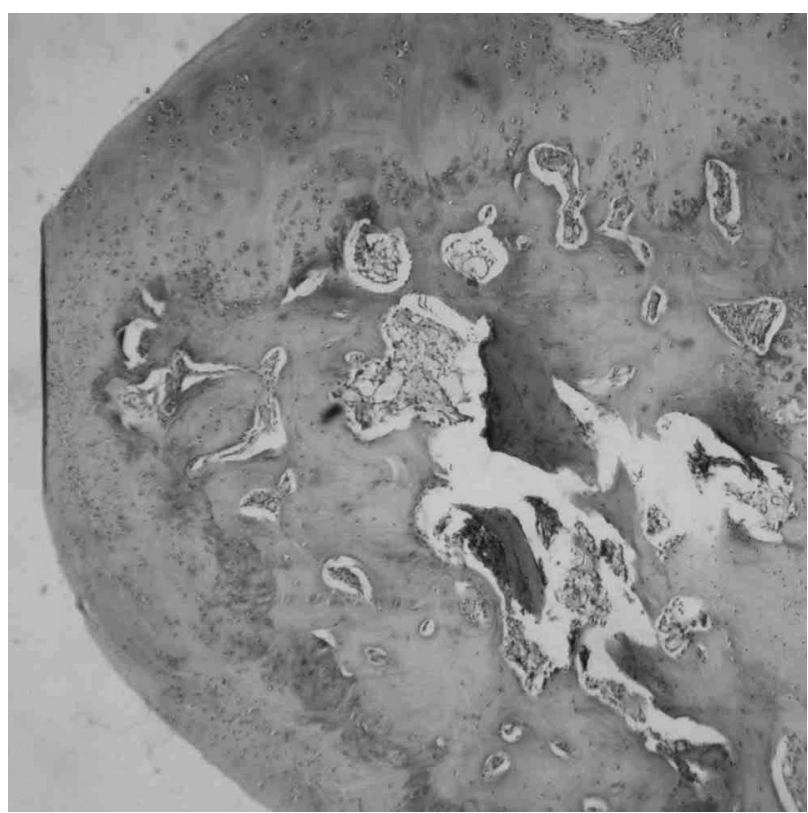

Fig. 6. Photomicrograph showing a thin, well formed cartilaginous cap with normal appearing chondrocytes over the normal bone and marrow $(\mathrm{H} \& \mathrm{E}, \times 40)$. increased intensity, indicating myelopathy (Fig. 3). The adjacent structures appeared to be normal. A skeletal survey for osteogenic tumors revealed no other lesions.

Posterior decompression with a laminectomy of $\mathrm{C} 1$ was undertaken. The right aspect of the posterior arch of $\mathrm{C} 1$ was removed slowly, allowing the intended dura to expand (Figs. 4 and 5). A histological examination disclosed mature bony tissue with a transition to proliferating cartilage, which formed a cap. The diagnosis was osteochondroma (Fig. 6).

The postoperative period was uneventful. Her JOA score had improved to 18, two-months after surgery. At the twoyear follow-up, she was symptom free without recurrence, and no instability in the flexion and extension radiograms was reported.

\section{Discussion}

Osteochondromas are common slow-growing benign bone tumors that are located frequently in the long bones, which are also known as "osteocartilaginous" or "benign exostosis." Approximately $1-4 \%$ of them occur in the spine [3]. Development in conjunction with HME, which is most often inherited as an autosomal dominant trait, is more frequent and has been reviewed thoroughly [2]. The solitary and multiple forms are detected in males more frequently than in females with a ratio of $1.8: 1$ (multiple) to $3: 1$ solitary [3]. Although it makes up approximately $11 \%$ of all benign bone tumors, its localization in the spine is observed in $<2$ $7 \%$ of cases [4].

Clinical manifestations in previously reported HME cases are in the second or third decades of life with a median age of 20 years [2]. An increased incidence of spinal cord compression has been reported in HME cases when compared to the solitary ones [3]. Few case reports in the literature describe a solitary osteochondroma in the absence of HME with spinal cord compression [5].

Solitary osteochondromas can arise from all parts of the spinal column with a predilection to the cervical area [1]. In the review report by Khosla et al. [5], there were 41 cases of solitary vertebral osteochondromas with spinal cord compression. Twenty-three cases (56\%) occurred in the cervical spine, 16 cases $(38 \%)$ in the thoracic spine, and two cases in the lumbar spine $(6 \%)$. The cervical spine has much greater mobility than any other part of the vertebral column, so it suffers from more microtraumas. This might cause a displacement of the epiphysial cartilage, which can then stimulate the tumor growth [6]. They can be located 
anywhere in the cervical spine, confluent with the marrow of the $\mathrm{C} 6$ articular pillar [7], spinous process of $\mathrm{C} 1$ [8], $\mathrm{C} 2$, $\mathrm{C} 6, \mathrm{C} 7$, from the neural arch of $\mathrm{C} 1$ [5] and $\mathrm{C} 2$ [8], from the anterior aspect of $\mathrm{C} 2$, and $\mathrm{C} 7$. Posterior elements can also be observed, which can be explained by numerous ossification centers of the neural arch that appear in the second decade of life. To the best of our knowledge, only two cases of solitary osteochondroma in the posterior arch of $\mathrm{C} 1$ with myelopathy have been reported [5].

Spinal osteochondromas can cause localized pain, restricted movement and headache when situated at the cervical level. Radiating symptoms and symptoms secondary to spinal stenosis, as shown in the present case, can also occur depending upon their relation to the vertebral canal and spinal canal, respectively $[5,8,9]$. The tumors increase in size by enchondral ossification of the cartilaginous cap. The symptom duration varies from less than one year to 13 years [1]. The interval between the first symptom and the development of myelopathy in the present case is long enough to challenge the hypothesis that the slow growing process of the tumor into the relatively larger canal at the $\mathrm{C} 1$ level may be one of the reasons for the delayed symptoms of myelopathy in the present patient.

For symptomatic patients, early diagnosis and treatment of the spinal osteochondromas are crucial before any neurological complications can occur. Surgeons do not need to be impatient for slow growing silent lesions. Plain roentgenograms can outline the typical sessile bone like projection of the tumor. Demonstration of the continuity between the bony cortex of the exostosis and the host bone is considered distinctive but is often not diagnostic [3]. The lesions appear as well-corticated masses with continuity of the central marrow with the adjacent normal osseous structures on CT. Unlike the typical long bone osteochondroma, spinal osteochondromas tend to be sessile rather than pedunculated [5]. The lesions show a prominent peripheral rim with low intensity corresponding to ossification and a small core with an intermediate signal similar to that of bone marrow, giving the mass a bull's-eye appearance $[4,5]$. A whole body scan may also be obtained to excluding multiple bone lesions.

Laminectomy with decompression of the neural elements is the treatment of choice for intracanalicular osteochondromas. Surgical therapy should consist of total removal of the osteochondroma, since an incomplete resection, leaving the cartilaginous cap inside, can cause a recurrence. Most operated patients showed improvement or the total disappearance of symptoms after the surgical resection [3].

Various tumors of the atlas may be considered in a differential diagnosis. In the literature, small series or individual cases harboring an isolated expanding lesion of the posterior arch of the atlas have been reported. Almost all these cases were osteogenic tumors including osteoblastomas and giant cell tumors. As an extremely rare cause, exuberant callus formation of the atlas has also been described [10]. A sudden increase in the size or the new onset of pain suggests malignant transformation, which occurs in approximately $1 \%$ of solitary and $10 \%$ of multiple osteochondromas [6]. Radiological manifestation of these lesions may challenge the diagnosis making a pathological examination essential for those patients.

Although spinal cord compression secondary to osteochondroma of the atlas lamina is an uncommon presentation, it must be considered in patients with persistent neck pain. The diagnosis and treatment of these tumors are of importance because they can cause spinal stenosis resulting in neural tissue compression. CT and MRI in conjunction with plain radiograms is the neuroradiological modality of choice. Surgical removal of the lesion is the preferred treatment before any neurological complications take place.

\section{REFERENCES}

1. Calhoun JM, Chadduck WM, Smith JL. Single cervical exostosis: report of a case and review of the literature. Surg Neurol 1992;37:26-9.

2. Labram EK, Mohan J. Diaphyseal aclasis with spinal cord compression: report of two cases and review of the literature. J Neurosurg 1996;84:518-21.

3. Albrecht S, Crutchfield JS, SeGall GK. On spinal osteochondromas. J Neurosurg 1992;77:247-52.

4. Prasad A, Renjen PN, Prasad ML, et al. Solitary spinal osteochondroma causing neural syndromes. Paraplegia 1992;30:678-80.

5. Khosla A, Martin DS, Awwad EE. The solitary intraspinal vertebral osteochondroma. An unusual cause of compressive myelopathy: features and literature review. Spine (Phila Pa 1976) 1999;24:77-81.

6. Baer-Henney S, Tatagiba M, Samii M. Osteochondroma of the cervical spine causing occipital nerve neuralgia: case report. Neurol Res 2001;23:777-9.

7. Eaton BA, Kettner NW, Essman JB. Solitary 
osteochondroma of the cervical spine. J Manipulative Physiol Ther 1995;18:250-3.

8. Cherubino P, Benazzo F, Castelli C. Osteochondroma of the cervical spine. Ital J Orthop Traumatol 1991;17:131-4.

9. Palmer FJ, Blum PW. Osteochondroma with spinal cord compression: report of three cases. J Neurosurg 1980;52:842-5.

10. Clavel M, Clavel P. Occipital neuralgia secondary to exuberant callus formation: case report. J Neurosurg 1996;85:1170-1. 